TRANSACTIONS OF THE

AMERICAN MATHEMATICAL SOCIETY

Volume 363, Number 12, December 2011, Pages 6257-6267

S 0002-9947(2011)05226-8

Article electronically published on June 27, 2011

\title{
A LOGARITHMIC DERIVATIVE LEMMA IN SEVERAL COMPLEX VARIABLES AND ITS APPLICATIONS
}

\author{
BAO QIN LI
}

\begin{abstract}
We give a logarithmic derivative lemma in several complex variables and its applications to meromorphic solutions of partial differential equations.
\end{abstract}

\section{INTRODUCTION}

The logarithmic derivative lemma of Nevanlinna is an important tool in the value distribution theory of meromorphic functions and its applications. It has two main forms (see [13, 1.3.3 and 4.2.1], 9, p. 115], 4, p. 36], etc.): Estimate (1) and its consequence, Estimate (2) below.

Theorem A. Let $f$ be a non-zero meromorphic function in $|z|<R \leq+\infty$ in the complex plane with $f(0) \neq 0, \infty$. Then for $0<r<\rho<R$,

$$
\begin{aligned}
& \frac{1}{2 \pi} \int_{0}^{2 \pi} \log ^{+}\left|\frac{f^{(k)}\left(r e^{i \theta}\right)}{f\left(r e^{i \theta}\right)}\right| d \theta \\
& \leq c\left\{\log ^{+} T(\rho, f)+\log ^{+} \log ^{+} \frac{1}{|f(0)|}+\log ^{+} \rho+\log ^{+} \frac{1}{\rho-r}+\log ^{+} \frac{1}{r}+1\right\},
\end{aligned}
$$

where $k$ is a positive integer and $c$ is a positive constant depending only on $k$.

Estimate (1) with $k=1$ was originally due to Nevanlinna, which easily implies the following version of the lemma with exceptional intervals of $r$ for meromorphic functions in the plane:

$$
\frac{1}{2 \pi} \int_{0}^{2 \pi} \log ^{+}\left|\frac{f^{\prime}\left(r e^{i \theta}\right)}{f\left(r e^{i \theta}\right)}\right| d \theta=O\{\log (r T(r, f))\},
$$

for all $r$ outside a countable union of intervals of finite Lebesgue measure, by using the Borel lemma in a standard way (see e.g. 4, [9, 13]).

Estimate (1) with $k>1$ was due to Hiong (see [5], 9, p. 115], [13, 4.2.1], etc.), which plays the same roles as Estimate (1) with $k=1$ when higher order derivatives are involved. It is simple to obtain Estimate (2) with $f^{\prime}$ replaced by $f^{(k)}$ by applying the result with $k=1$ to each factor in the product $\frac{f^{(k)}}{f}=\frac{f^{(k)}}{f^{(k-1)}} \frac{f^{(k-1)}}{f^{(k-2)}} \cdots \frac{f^{\prime}}{f}$. However, it should be noted that Estimate (1) with $k>1$ (Hiong's result) can not be obtained from Estimate (1) with $k=1$ (Nevanlinna's original lemma) by applying Estimate (1) with $k=1$ to each factor of the above product, as one might

Received by the editors January 8, 2009 and, in revised form, September 23, 2009.

2010 Mathematics Subject Classification. Primary 32A22, 32A20, 30D35.

(C)2011 American Mathematical Society 
be tempted to do, since it would give rise to extra terms involving $\log ^{+} \log ^{+} \frac{1}{\left|f^{(j)}(0)\right|}$ $(1 \leq j \leq k-1)$

While one often sees the uses of Estimate (2) in questions where exceptional intervals of $r$ are allowed and only large values of $r$ are concerned, the more precise Estimate (1) is needed when more precise local information of functions is required. Some questions on normal families in $\mathbf{C}^{n}$ and related problems led us to ask whether the same estimate in Theorem A holds for meromorphic functions in $\mathbf{C}^{n}$. Note that the $\mathbf{C}^{n}$-version of Estimate (2) was proved by Vitter in [12] and another proof was given in 2]; see also [14] for a related estimate. However, none of these estimates can yield the estimate in Theorem A. We emphasize that the estimate in Theorem A holds without exceptional intervals and involves only two terms $\log ^{+} \log ^{+} \frac{1}{|f(0)|}$ and $\log ^{+} T(\rho, f)$ that depend on the given function $f$, which may make a significant difference from other estimates, since any other terms may require further delicate estimates or may be impossible to estimate in practice. (This point is made clear in the proofs of Theorem 2 and Corollary 3 in $\S 3$. )

In this paper, we will show that exactly the same Estimate (1) in Theorem A also holds in $\mathbf{C}^{n}$ (Theorem 1 below). The proof is short and works for the cases $k=1$ and $k>1$ at the same time. The result easily implies the $\mathbf{C}^{n}$-version of Estimate (2) in a standard way.

While generalizing Theorem A to several variables is a natural goal, it is hoped that Theorem 1 would become a useful tool in several complex variables beyond Nevanlinna theory, as Theorem A in one complex variable. An application of Theorem 1 to meromorphic solutions of partial differential equations is given in the last section of the paper.

\section{ThE LOGARITHMIC DERIVATIVE LEMMA}

We will assume familiarity with the basics of Nevanlinna theory such as the counting function $N(r, f)$ and the Nevanlinna characteristic $T(r, f):=m(r, f)+$ $N(r, f)$, where $m(r, f)=\int_{S_{n}(r)} \log ^{+}|f| \eta_{n}$ for a meromorphic function $f$ in $|z|<$ $R \leq \infty$ in $\mathbf{C}^{n}, \eta_{n}=d^{c} \log |z|^{2} \wedge\left(d d^{c} \log |z|^{2}\right)^{n-1}$, a positive measure on the sphere $S_{n}(r):=\left\{z \in \mathbf{C}^{n}:|z|=r\right\}$ with total measure 1. We will also use $\sigma_{n}=\left(d d^{c}|z|^{2}\right)^{n}$, the Lebesque measure on the ball $B_{n}(r)=\left\{z \in \mathbf{C}^{n}:|z| \leq r\right\}$ normalized such that the total volume of $B_{n}(r)$ is $r^{2 n}$. (We omit the explicit definition of the counting function, which is not needed in the paper.) For convenience we will also use the slightly different characteristic (cf. [10, pp. 17-18])

$$
T_{f}(r):=\int_{S_{n}(r)} \log \sqrt{|g|^{2}+|h|^{2}} \eta_{n}-\log \sqrt{|g(0)|^{2}+|h(0)|^{2}},
$$

where $g$ and $h$ are two coprime holomorphic functions such that $f=\frac{g}{h}$ if $f(0)$ is finite. In the one-variable case, $T_{f}(r)$ is the Ahlfors-Shimizu characteristic. If $f(0)$ is finite, by the Jensen formula, (3) can also be written as

$$
T_{f}(r):=\int_{S_{n}(r)} \log \sqrt{1+|f|^{2}} \eta_{n}+N(r, f)-\log \sqrt{1+|f(0)|^{2}} .
$$

It is clear that

$$
\begin{aligned}
& T(r, f) \leq T_{f}(r)+\log \sqrt{1+|f(0)|^{2}} \\
& T_{f}(r) \leq T(r, f)-\log \sqrt{1+|f(0)|^{2}}+\frac{1}{2} \log 2 .
\end{aligned}
$$


Also note the basic properties of $\log ^{+} x$ : for $a_{j} \geq 0$ and integrable $h \geq 0$ on $S_{n}(r)$ or $B_{n}(r)$,

$$
\log ^{+} \prod_{j=1}^{m} a_{j} \leq \sum_{j=1}^{m} \log ^{+} a_{j}, \quad \log ^{+} \sum_{j=1}^{m} a_{j} \leq \sum_{j=1}^{m} \log ^{+} a_{j}+\log m,
$$

and by the concavity of $\log x$,

$$
\begin{aligned}
& \int_{S_{n}(r)} \log ^{+} h \eta_{n} \leq \int_{S_{n}(r)} \log (h+1) \eta_{n} \\
& \leq \log \int_{S_{n}(r)}(h+1) \eta_{n} \leq \log ^{+} \int_{S_{n}(r)} h \eta_{n}+\log 2 ;
\end{aligned}
$$

similarly,

$$
\int_{B_{n}(r)}\left(\log ^{+} h\right) \frac{\sigma_{n}}{r^{2 n}} \leq \log ^{+} \int_{B_{n}(r)} h \frac{\sigma_{n}}{r^{2 n}}+\log 2 .
$$

We will use the following.

Lemma B ([10, p. 19]). Suppose that $f$ is meromorphic in $|z|<R \leq+\infty$ in $\mathbf{C}^{n}$ and $H$ is a linear subspace of $\mathbf{C}^{n}$. Then for $0<r<\rho<R$,

$$
T_{f \mid H}(r) \leq \frac{1+\theta}{(1-\theta)^{2 n-1}} T_{f}\left(\frac{r}{\theta}\right)
$$

if $0<\theta<1$ with $\frac{r}{\theta}<R$ and $f$ is holomorphic at 0 , where $f \mid H$ is the restriction of $f$ to $H$. In particular, taking $\theta=\frac{r}{\rho}$, then

$$
T_{f \mid H}(r) \leq \frac{2 \rho^{2 n-1}}{(\rho-r)^{2 n-1}} T_{f}(\rho) .
$$

We will also use the following elementary result about integration on $S_{n}(r)$, which reduces integration on the sphere to one-variable integration ([2, p. 35]; also see [11]): If $h$ is a function such that $h \eta_{n}$ is integrable on $S_{n}(r)$, then

$$
\int_{S_{n}(r)} h \eta_{n}=r^{2-2 n} \int_{B_{n-1}(r)}\left(\int_{S_{1}\left(\sqrt{r^{2}-|w|^{2}}\right)} h_{w}\left(z_{n}\right) \eta_{1}\left(z_{n}\right)\right) \sigma_{n-1}(w),
$$

where $w=\left(z_{1}, z_{2}, \cdots, z_{n-1}\right) \in \mathbf{C}^{n-1}$ and $h_{w}\left(z_{n}\right)=h\left(w, z_{n}\right)$ is the restriction of $h$ to the variable $z_{n}$.

We give our generalization of Theorem A.

Theorem 1. Let $f$ be a non-zero meromorphic function in $|z|<R \leq+\infty$ in $\mathbf{C}^{n}$ with $f(0)$ being a non-zero complex number and $k$ a positive integer. Then for $0<r<\rho<R$ and $1 \leq j \leq n$,

$$
\begin{aligned}
& \int_{S_{n}(r)} \log ^{+}\left|\frac{\frac{\partial^{k} f}{\partial z_{j}^{k}}}{f}\right| \eta_{n} \\
& \leq c\left\{\log ^{+} T(\rho, f)+\log ^{+} \log ^{+} \frac{1}{|f(0)|}+\log ^{+} \rho+\log ^{+} \frac{1}{\rho-r}+\log ^{+} \frac{1}{r}+1\right\},
\end{aligned}
$$

where $c$ is a positive constant depending only on $k$ and $n$.

The estimate in Theorem 1 easily implies the $\mathbf{C}^{n}$-version of Estimate (2) in [12] by the Borel Lemma. 
Proof of Theorem 1. Without loss of generality, take $j=n$. Let

$$
F(z):=f\left(z_{1}, \cdots, z_{n-1}, 0\right)=f(w, 0), \quad f_{w}\left(z_{n}\right):=f\left(w, z_{n}\right),
$$

where $w=\left(z_{1}, \cdots, z_{n-1}\right) \in \mathbf{C}^{n-1}, z_{n} \in \mathbf{C}$. Then $F(0)=f(0)$. Since $f(0)$ is a non-zero complex number, $f(w, 0) \not \equiv 0, \infty$ for $w \in \mathbf{C}^{n-1}$, which implies that the set of $w$ where $f(w, 0)=0$ or $\infty$ is a thin set in $\mathbf{C}^{n-1}$. Thus, $f_{w}$ is a meromorphic function of $z_{n}$ with $f_{w}(0)=f(w, 0) \neq 0, \infty$ for all $w \in B_{n-1}(r) \subset \mathbf{C}^{n-1}$ except a thin set, which is of Lebesgue measure zero in $B_{n-1}(r)$. Write $f=\frac{g}{h}$, where $g$ and $h$ are two coprime holomorphic functions in $|z|<R$. Denote $\|f\|=\sqrt{|g|^{2}+|h|^{2}}$, and denote $\left\|f_{w}\right\|$ and $\|F\|$ in the same way. By formula (6) and Theorem A, we have that for $0<r<\rho<R$,

$$
\begin{aligned}
& \int_{S_{n}(r)} \log ^{+}\left|\frac{\partial^{k} f}{\partial z_{n}^{k}} / f\right| \eta_{n} \\
& =r^{2-2 n} \int_{B_{n-1}(r)}\left(\int_{S_{1}\left(\sqrt{r^{2}-|w|^{2}}\right)} \log ^{+}\left|\frac{f_{w}^{(k)}\left(z_{n}\right)}{f}\right| \eta_{1}\left(z_{n}\right)\right) \sigma_{n-1}(w) \\
& \leq c_{0} r^{2-2 n} \int_{B_{n-1}(r)}\left\{\log ^{+} T\left(\sqrt{\rho^{2}-|w|^{2}}, f_{w}\right)+\log ^{+} \log ^{+} \frac{1}{\left|f_{w}(0)\right|}+\log ^{+} \rho\right. \\
& \left.\quad+\log ^{+} \frac{1}{\sqrt{\rho^{2}-|w|^{2}}-\sqrt{r^{2}-|w|^{2}}}+\log ^{+} \frac{1}{\sqrt{r^{2}-|w|^{2}}}+1\right\} \sigma_{n-1}(w),
\end{aligned}
$$

where $c_{0}$ is a constant depending only on $k$.

We now estimate each of the terms on the right-hand side of (7). By (6), (4), and the concavity of $\log x$ (see $\S 2$ ), we have that

$$
\begin{aligned}
& r^{2-2 n} \int_{B_{n-1}(r)} \log ^{+} \log ^{+} \frac{1}{\left|f_{w}(0)\right|} \sigma_{n-1}=\int_{S_{n}(r)} \log ^{+} \log ^{+} \frac{1}{|F|} \eta_{n} \\
& \leq \log ^{+} \int_{S_{n}(r)} \log ^{+} \frac{1}{|F|} \eta_{n}+\log 2 \leq \log ^{+} T\left(r, \frac{1}{F}\right)+\log 2 \\
& \leq \log ^{+}\left\{T_{F}(r)+\log \sqrt{1+\frac{1}{|F(0)|^{2}}}\right\}+\log 2 \\
& \leq \log ^{+}\left\{T_{F}(r)+\log ^{+} \frac{1}{|f(0)|}+\frac{1}{2} \log 2\right\}+\log 2 \\
& \leq \log ^{+} T_{F}(r)+\log ^{+} \log ^{+} \frac{1}{|f(0)|}+\log 6 \\
& \leq \log ^{+}\left\{\frac{2 \rho^{2 n-1}}{(\rho-r)^{2 n-1}} T_{f}(\rho)\right\}+\log { }^{+} \log +\frac{1}{|f(0)|}+\log 6 \\
& \leq \log ^{+}\left\{\frac{2 \rho^{2 n-1}}{(\rho-r)^{2 n-1}}\left(T(\rho, f)+\frac{1}{2} \log 2\right)\right\}+\log ^{+} \log ^{+} \frac{1}{|f(0)|}+\log 6,
\end{aligned}
$$

using (5) with $f \mid H=F$ in Lemma B.

By (4) and (3), we have that

$$
\begin{aligned}
& T\left(\sqrt{\rho^{2}-|w|^{2}}, f_{w}\right) \leq T_{f_{w}}\left(\sqrt{\rho^{2}-|w|^{2}}\right)+\log \sqrt{1+\left|f_{w}(0)\right|^{2}} \\
& =\int_{S_{1}\left(\sqrt{\rho^{2}-|w|^{2}}\right)} \log \| f_{w}|| \eta_{1}-\log || f_{w}(0)||+\log \sqrt{1+\left|f_{w}(0)\right|^{2}} .
\end{aligned}
$$


Using this inequality, the concavity of $\log x$ (see $\S 2$ ), and (6), we deduce that

$$
\begin{aligned}
& r^{2-2 n} \int_{B_{n-1}(r)} \log ^{+} T\left(\sqrt{\rho^{2}-|w|^{2}}, f_{w}\right) \sigma_{n-1}(w) \\
& \leq r^{2-2 n} \int_{B_{n-1}(\rho)} \log ^{+}\left(\int_{S_{1}\left(\sqrt{\rho^{2}-|w|^{2}}\right)} \log \left\|f_{w}\right\| \eta_{1}-\log \left\|f_{w}(0)\right\|\right. \\
& \left.\quad+\log \sqrt{1+\left|f_{w}(0)\right|^{2}}\right) \sigma_{n-1}(w) \\
& \leq \log ^{+}\left\{( \frac { \rho } { r } ) ^ { 2 n - 2 } \rho ^ { 2 - 2 n } \int _ { B _ { n - 1 } ( \rho ) } \left(\int_{S_{1}\left(\sqrt{\rho^{2}-|w|^{2}}\right)} \log \left\|f_{w}\right\| \eta_{1}-\log \left\|f_{w}(0)\right\|\right.\right. \\
& \left.\left.\quad+\log \sqrt{1+\left|f_{w}(0)\right|^{2}}\right) \sigma_{n-1}(w)\right\}+\log 2 \\
& =\log ^{+}\left\{( \frac { \rho } { r } ) ^ { 2 n - 2 } \left(\int_{S_{n}(\rho)} \log \|f\| \eta_{n}-\int_{S_{n}(\rho)} \log \|F\| \eta_{n}\right.\right. \\
& \left.\left.\quad+\int_{S_{n}(\rho)} \log \sqrt{1+|F|^{2}} \eta_{n}\right)\right\}+\log 2 \\
& \leq \log ^{+}\left\{\int_{S_{n}(\rho)} \log \left\|f|| \eta_{n}-\int_{S_{n}(\rho)} \log \right\| F|| \eta_{n}+\int_{S_{n}(\rho)} \log \sqrt{1+|F|^{2}} \eta_{n}\right\} \\
& \quad+(2 n-2)\left(\log ^{+} \rho+\log ^{+} \frac{1}{r}\right)+\log 2 .
\end{aligned}
$$

By (3) we have that

$$
\int_{S_{n}(\rho)} \log \|f\| \eta_{n}=T_{f}(\rho)+\log \|f(0)\|, \quad \int_{S_{n}(\rho)} \log \|F\| \eta_{n}=T_{F}(\rho)+\log \|F(0)\| .
$$

Also, by (4),

$$
\begin{aligned}
& \int_{S_{n}(\rho)} \log \sqrt{1+|F|^{2}} \eta_{n} \leq \int_{S_{n}(\rho)} \frac{1}{2} \log ^{+}\left(1+|F|^{2}\right) \eta_{n} \\
& \leq \int_{S_{n}(\rho)} \log ^{+}|F| \eta_{n}+\frac{1}{2} \log 2 \leq T(\rho, F)+\frac{1}{2} \log 2 \\
& \leq T_{F}(\rho)+\log \sqrt{1+|F(0)|^{2}}+\frac{1}{2} \log 2 .
\end{aligned}
$$

We thus obtain that

$$
\begin{aligned}
& \log ^{+}\left\{\int_{S_{n}(\rho)} \log \|f\| \eta_{n}-\int_{S_{n}(\rho)} \log \|F\| \eta_{n}+\int_{S_{n}(\rho)} \log \sqrt{1+|F|^{2}} \eta_{n}\right\} \\
& \leq \log ^{+}\left\{T_{f}(\rho)+\log \|f(0)\|-\left(T_{F}(\rho)+\log \|F(0)\|\right)+T_{F}(\rho)\right. \\
& \left.\quad+\log \sqrt{1+|F(0)|^{2}}+\frac{1}{2} \log 2\right\} \\
& =\log ^{+}\left\{T_{f}(\rho)+\log \sqrt{1+|F(0)|^{2}}+\frac{1}{2} \log 2\right\} \\
& \leq \log ^{+}\left\{T(\rho, f)-\log \sqrt{1+|f(0)|^{2}}+\log \sqrt{1+|F(0)|^{2}}+\log 2\right\} \\
& \leq \log ^{+} T(\rho, f)+\log 2 .
\end{aligned}
$$


Also, it is easy to verify that $\frac{1}{\sqrt{\rho^{2}-|w|^{2}}-\sqrt{r^{2}-|w|^{2}}} \leq \frac{1}{\rho-r}$ and that

$$
\begin{aligned}
& r^{2-2 n} \int_{B_{n-1}(r)} \log ^{+} \frac{1}{\sqrt{r^{2}-|w|^{2}}} \sigma_{n-1} \\
& =\int_{B_{n-1}(1)} \log ^{+} \frac{1}{r \sqrt{1-|w|^{2}}} \sigma_{n-1} \leq \log ^{+} \frac{1}{r}+c_{1}
\end{aligned}
$$

with $c_{1}=\int_{B_{n-1}(1)} \log ^{+} \frac{1}{\sqrt{1-|w|^{2}}} \sigma_{n-1}$.

Combining the above estimates we obtain from (7) that

$$
\begin{gathered}
\int_{S_{n}(r)} \log ^{+}\left|\frac{\partial^{k} f}{\partial z_{n}^{k}} / f\right| \eta_{n} \leq c_{0}\left\{2 \log ^{+} T(\rho, f)+\log ^{+} \log ^{+} \frac{1}{|f(0)|}\right. \\
\left.+(4 n-2) \log ^{+} \rho+2 n \log ^{+} \frac{1}{\rho-r}+(2 n-1) \log ^{+} \frac{1}{r}+c_{2}\right\} \\
\leq c\left\{\log ^{+} T(\rho, f)+\log ^{+} \log ^{+} \frac{1}{|f(0)|}+\log ^{+} \rho+\log ^{+} \frac{1}{\rho-r}+\log ^{+} \frac{1}{r}+1\right\},
\end{gathered}
$$

where $c_{2}=1+5 \log 2+\log 3+c_{1}$, and $c$ is a positive constant depending only on $k$ and $n$. This proves the theorem.

\section{Applications}

As applications of Theorem 1, we give in this section a Schottky-type estimate and a Montel-type normality criterion for meromorphic solutions of the partial differential equations (8) below. The proofs of the results adopt the estimate in Theorem 1 for both order $k=1$ and order $k>1$, from which it is clear why the dependence of the estimate on $\log ^{+} T(\rho, f)$ and $\log ^{+} \log ^{+} \frac{1}{|f(0)|}$ only and without exceptional intervals may be important.

Consider the partial differential equations

$$
L(y):=L\left(z, y, \frac{\partial y}{\partial z_{1}}, \cdots, \frac{\partial y}{\partial z_{n}}, \cdots\right)=b(z)
$$

in $\mathbf{C}^{n}$, where $L$ is a homogeneous polynomial of degree $q \geq 1$ in $y$ and all partial derivatives $\frac{\partial y}{\partial z_{1}}, \cdots, \frac{\partial y}{\partial z_{n}}, \cdots$ of order at most $m$ with polynomial coefficients, and $b \not \equiv 0$ is a polynomial. The special case with $q=1$ is $L=\sum_{|I|=0}^{m} a_{I} \frac{\partial^{|I|} y}{\partial^{i n} z_{n} \cdots \partial^{i 1} z_{1}}$, where $I=\left(i_{1}, \cdots, i_{n}\right) \in\left(\mathbf{Z}^{+}\right)^{n}$ with $|I|=i_{1}+\cdots+i_{n}, \mathbf{Z}^{+}=\{0,1,2, \cdots\}$, and the $a_{I}$ 's are polynomials. In this case, equation (8) is a general non-homogeneous linear partial differential equation with polynomial coefficients. The well-known eiconal (eikonal) partial differential equations $\left(\frac{\partial u}{\partial z_{1}}\right)^{2}+\left(\frac{\partial u}{\partial z_{2}}\right)^{2}+\cdots+\left(\frac{\partial u}{\partial z_{n}}\right)^{2}=1$ and the Fermat partial differential equations $\left(\frac{\partial u}{\partial z_{1}}\right)^{q}+\left(\frac{\partial u}{\partial z_{2}}\right)^{q}+\cdots+\left(\frac{\partial u}{\partial z_{n}}\right)^{q}=1(q \geq 3)$ are all special cases of (8).

Theorem 2. Let $f$ be a meromorphic solution of (8) in the ball $|z|<R<+\infty$ of $\mathbf{C}^{n}$. If $f(z) \neq 0$ in the ball, then for $0<r<R$,

$$
T(r, f) \leq c\left\{1+\log ^{+}|f(0)|+\log ^{+} R+\log ^{+} \frac{1}{R-r}\right\},
$$

where $c$ is a positive constant independent of $f, r, R$. 
The estimate in Theorem 2 is motivated by the famous Schottky theorem (see e.g. [4], 9], 13]), which estimates the maximum modulus of a holomorphic function $f$ that omits 0 and 1 in $|z|<R$ with a similar bound in terms of $f(0), R$ and $r<R$ : $\log \max _{|z|=r}|f(z)| \leq \frac{c R}{R-r}\left(\log ^{+}|f(0)|+\log \frac{2 R}{R-r}\right)$. The function $f$ is required to omit 0 and 1. It is this condition that produces a series of important theorems including the Montel normality criterion (see below), thereby constituting the socalled Montel cycle. Theorem 2 is concerned with solutions $f$ of partial differential equations of the form (8) with homogeneity and we are thus able to require less: $f$ is allowed to be meromorphic, and is only required to omit 0 . This condition of course cannot be dropped (cf. Remark 4(i) below).

As an application of Theorem 2, we have the following.

Corollary 3. A family of meromorphic solutions of (8) in a domain $D$ of $\mathbf{C}^{n}$ that omits 0 is a normal family in $D$.

Corollary 3 is in the direction of the celebrated Montel normality criterion (see e.g. [1] [9], [13]): A family of holomorphic functions in a domain $D$ of the complex plane that omits 0 and 1 is a normal family in $D$ (the meromorphic version requires that the functions omit three distinct values). The notion of a normal family has played an important role in the development of complex function theory. We refer to [1], 3], 6], 9], 13, etc. and the references therein for various results on normal families and applications.

Remark 4. We address a number of natural questions raised by Theorem 2 to show that the result is "best possible".

(i) As mentioned above, the condition that $f$ omits 0 in Theorem 2 cannot be dropped. For example, the entire function $u=z_{1}+e^{z_{2}-z_{3}}$, which clearly has zeros, is a solution of the Fermat partial differential equation $\left(\frac{\partial u}{\partial z_{1}}\right)^{q}+\left(\frac{\partial u}{\partial z_{2}}\right)^{q}+\left(\frac{\partial u}{\partial z_{3}}\right)^{q}=1(q$ is odd). The solution $u$ cannot satisfy the estimate in Theorem 2 , since otherwise $T(r, u)=O(\log r)$ (by taking $R=2 r$ in Theorem 2), which implies that $u$ is a polynomial, a contradiction.

(ii) It is assumed that $b(z) \not \equiv 0$ in (8). When $q=1$, this means that the linear partial differential equation is non-homogeneous. Can this condition be removed? Does Theorem 2 also hold for homogeneous linear partial differential equations, i.e., $b(z) \equiv 0$ ? The answer is negative. For example, $u=e^{z_{1}-z_{2}}$ is an entire solution of the equation $\left(\frac{\partial u}{\partial z_{1}}\right)^{q}+\left(\frac{\partial u}{\partial z_{2}}\right)^{q}=0$ ( $q$ is odd), which is of the form (8) with, however, $b(z)=0$. The solution $u$ omits 0 , but $u$ cannot satisfy the estimate in Theorem 2 , since otherwise $u$ would be a polynomial, as in (i).

(iii) It is trivial to note that a meromorphic function omitting 0 but without being a solution of (8) in general cannot satisfy the estimate in Theorem 2 (see the example in (ii)).

Remark 5. As in Remark 4 for Theorem 2, let us show that the result in Corollary 3 is also "best possible".

(i) The condition that $f$ omits 0 cannot be dropped in Corollary 3. For example, consider the sequence $\left\{f_{n}\right\}$ with $f_{n}\left(z_{1}, z_{2}\right)=e^{n z_{1}+z_{2}}-1$, which is clearly not a normal family in $|z|<1$, since $f_{n}(0,0)=0$ but $f_{n}\left(\frac{1}{2}, 0\right) \rightarrow \infty$. But each $f_{n}$ is a solution of the equation $\frac{\partial w}{\partial z_{2}}-w=1$ of the form (8). It is easy to check that $f_{n}(z)$ has zeros in any neighborhood of the origin for large $n$.

(ii) The condition that meromorphic functions in a family do not assume 0 alone (without being solutions) is of course not enough for normality in Corollary 3. For 
example, $\left\{f_{n}\right\}$ with $f_{n}(z)=\frac{1}{1-e^{n z}}$ is clearly not normal in $|z|<1$, although $\frac{1}{1-e^{n z}}$ omits 0 (it also omits 1). By Montel's theorem mentioned above, it in general requires that the meromorphic functions do not assume three distinct values.

(iii) As in Theorem 2, the condition $b(z) \not \equiv 0$ in (8) cannot be dropped in Corollary 3. Consider $f_{n}\left(z_{1}, z_{2}\right)=e^{n z_{1}+z_{2}}$. Then $f_{n}(z) \neq 0$ and $f_{n}$ is a solution of $\frac{\partial w}{\partial z_{2}}-w=0$ with $b(z)=0$. But, $\left\{f_{n}\right\}$ is clearly not normal in $|z|<1$.

Proof of Theorem 2. Since

$$
L(f)=L\left(z, f, \frac{\partial f}{\partial z_{1}}, \cdots, \frac{\partial f}{\partial z_{n}}, \cdots\right)=b(z),
$$

we have that $\frac{b}{f^{q}}=\frac{L(f)}{f^{q}}$, where $q$ is the degree given in (8). We may assume that $f(0) \neq \infty$, since otherwise the estimate in the theorem has $+\infty$ on the right-hand side and is already true. Applying Theorem 1 (for orders ranging from 0 to $\mathrm{m}$ ) to each term in $\frac{L(f)}{f^{q}}$ and noting that for any polynomial $a$,

$$
T(r, a) \leq c_{1}(1+\log r)
$$

for some $c_{1}>0$, we have that for $0<r<\rho<R$,

$$
\begin{aligned}
& m\left(r, \frac{1}{f}\right)=\frac{1}{q} m\left(r, \frac{1}{f^{q}}\right) \leq \frac{1}{q}\left\{m\left(r, \frac{b}{f^{q}}\right)+m\left(r, \frac{1}{b}\right)\right\}=\frac{1}{q}\left\{m\left(r, \frac{L(f)}{f^{q}}\right)+m\left(r, \frac{1}{b}\right)\right\} \\
& \leq c_{2}\left\{\log ^{+} T(\rho, f)+\log ^{+} \log ^{+} \frac{1}{|f(0)|}+\log ^{+} \rho+\log ^{+} \frac{1}{\rho-r}+\log ^{+} \frac{1}{r}+1\right\} \\
& +c_{3}(1+\log r),
\end{aligned}
$$

where $c_{2}$ is a positive constant depending only on $n, m$ and $q$ in (8), and $c_{3}$ is a positive constant depending only on $b(z)$ and the polynomial coefficients in (8). We may assume that $c_{2} \geq e$. It is easy to check that for any $x>0$ and $A \geq e$,

$$
\log x+A \log ^{+} \log ^{+} \frac{1}{x} \leq \log ^{+} x+A(\log A-1)
$$

(see e.g. [13, p. 47]). Thus,

$$
\log |f(0)|+c_{2} \log ^{+} \log ^{+} \frac{1}{|f(0)|} \leq \log ^{+}|f(0)|+c_{2}\left(\log c_{2}-1\right) .
$$

Since $f(z) \neq 0$, we have that, by virtue of (9) and (10),

$$
\begin{aligned}
& T(r, f)=T\left(r, \frac{1}{f}\right)+\log |f(0)|=m\left(r, \frac{1}{f}\right)+\log |f(0)| \\
& \leq \log ^{+}|f(0)|+c_{2}\left(\log c_{2}-1\right)+c_{2}\left\{\log ^{+} T(\rho, f)+\log ^{+} \rho\right. \\
& \left.\quad+\log ^{+} \frac{1}{\rho-r}+\log ^{+} \frac{1}{r}+1\right\}+c_{3}(1+\log r) \\
& \leq \log ^{+}|f(0)|+c_{2}\left(\log c_{2}-1\right)+c_{2}\left\{\log ^{+} R+\log ^{+} \frac{1}{r}+1\right\}+c_{3}(1+\log R) \\
& \quad+c_{2} \log ^{+} T(\rho, f)+c_{2} \log ^{+} \frac{1}{\rho-r} .
\end{aligned}
$$

We then use the following Bureau lemma (see [5, [13, p. 48]): Let $a(r)$ be a nonnegative and non-increasing function in $(0, R)$, and $b$ and $c$ two constants. If for $0<r<\rho<R$,

$$
T(r, f)<a(r)+b \log ^{+} \frac{1}{\rho-r}+c \log ^{+} T(\rho, f),
$$


then

$$
T(r, f)<2 a(r)+B \log ^{+} \frac{2}{R-r}+C
$$

for $0<r<R$, where $B$ and $C$ depend only on $b$ and $c$. Applying this result to our situation, we obtain that

$$
T(r, f) \leq c_{4}\left\{1+\log ^{+}|f(0)|+\log ^{+} \frac{1}{r}+\log ^{+} R+\log ^{+} \frac{1}{R-r}\right\},
$$

where $c_{4}$ is a constant depending only on $c_{2}$ and $c_{3}$. Thus, when $\frac{R}{2} \leq r<R$, we have that

$$
\begin{aligned}
& T(r, f) \leq c_{4}\left\{1+\log ^{+}|f(0)|+\log ^{+} \frac{2}{R}+\log ^{+} R+\log ^{+} \frac{1}{R-r}\right\} \\
& \leq c\left\{1+\log ^{+}|f(0)|+\log ^{+} R+\log ^{+} \frac{1}{R-r}\right\}
\end{aligned}
$$

where $c$ is a positive constant depending only on $n, m, q, b(z)$ and the polynomial coefficients in (8). This is also true for $r \leq \frac{R}{2}$, since for such an $r$ we have that $T(r, f) \leq T\left(\frac{R}{2}, f\right)$, which gives the same bound.

Proof of Corollary 3. Let $F$ be a family of meromorphic solutions of (8) in a domain $D$ of $\mathbf{C}^{n}$ that do not assume 0 . Since normality is a local property, we only need to show that $F$ is a normal family in a neighborhood of each point $\zeta$ in the domain $D$. Take a small ball $\{|z-\zeta|<R\} \subset D$ and any sequence $\left\{f_{l}\right\}$ in $F$. For any fixed $l$, we write $f=f_{l}$ for notational convenience. We claim that either $|f(z)| \leq 1$ for all $z$ in the ball $|z-\zeta|<\frac{R}{16}$, or $\frac{1}{|f(z)|} \leq c$ for all $z$ in the ball $|z-\zeta|<\frac{R}{16}$, where $c$ is a constant independent of $l$ and $z$. If $|f(z)| \leq 1$ for all $z$ in $|z-\zeta|<\frac{R}{16}$, or $|f(z)| \geq 1$ for all $z$ in $|z-\zeta|<\frac{R}{16}$, the claim then already holds in this case. In the opposite case, we have $|f(z)| \leq 1$ for some $z$ and $|f(z)| \geq 1$ for some other $z$ in $|z-\zeta|<\frac{R}{16}$. Then, by the continuity of $|f(z)|$ (outside the set of singularities, which has measure zero), we obtain a point $w$ in $|z-\zeta|<\frac{R}{16}$ such that $|f(w)|=1$. Applying Theorem 2 for $f$ in the ball $|z-w|<\frac{R}{2}$ (i.e., for $g(z):=f(w+z)$ in the ball $|z|<\frac{R}{2}$ ), we have that for $0<r<\frac{R}{2}$,

$$
\begin{aligned}
& T(r, w, f) \leq c\left\{1+\log ^{+}|f(w)|+\log ^{+} \frac{R}{2}+\log ^{+} \frac{R}{R-2 r}\right\} \\
& =c\left\{1+\log ^{+} \frac{R}{2}+\log ^{+} \frac{R}{R-2 r}\right\},
\end{aligned}
$$

where $T(r, w, f)$ is the characteristic of $f$ in the ball $|z-w|<\frac{R}{2}$ and $c$ is a constant independent of $l$ and $z$. We can assume that $c \geq 1$. We then have that for $0<r<\frac{R}{2}$,

$$
\begin{aligned}
& T\left(r, w, \frac{1}{f}\right)=T(r, w, f)+\log \frac{1}{f(w)} \\
& =T(r, w, f) \leq c\left\{1+\log ^{+} \frac{R}{2}+\log ^{+} \frac{R}{R-2 r}\right\} .
\end{aligned}
$$

Since $f \neq 0$ in $|z-\zeta|<R, \frac{1}{f}$ is holomorphic in $|z-\zeta|<R$. We can then use the following result (see e.g. [10, p. 21]): For any holomorphic function $g$ in $|z|<R$ in $\mathbf{C}^{n}$,

$$
\log ^{+} M(\theta r, g) \leq \frac{1+\theta}{(1-\theta)^{2 n-1}}\left(T(r, g)+\frac{\log 2}{2}\right)
$$


for $0<\theta<1$ and $0<r<R$, where $M(r, g)=\max _{|z|=r}\{|f(g)|\}$. Applying this result with $g=\frac{1}{f}$ in $|z-w|<\frac{R}{2}$ and $\theta=\frac{1}{2}, r=\frac{R}{4}$, we obtain that

$$
\begin{aligned}
& \log ^{+} M\left(\frac{R}{8}, w, \frac{1}{f}\right) \leq 3\left(2^{2 n-2}\right)\left\{T\left(\frac{R}{4}, w, \frac{1}{f}\right)+\frac{\log 2}{2}\right\} \\
& \leq 3 c 2^{2 n-2}\left\{1+\frac{3}{2} \log 2+\log ^{+} \frac{R}{2}\right\} .
\end{aligned}
$$

Noting that the ball $|z-\zeta|<\frac{R}{16}$ is contained in the ball $|z-w|<\frac{R}{8}$, we thus obtain that in $|z-\zeta|<\frac{R}{16}$,

$$
\frac{1}{|f(z)|} \leq 3 c 2^{2 n-2}\left\{1+\frac{3}{2} \log 2+\log ^{+} \frac{R}{2}\right\} .
$$

This proves the claim.

From the above claim, we see that in $|z-\zeta|<\frac{R}{16}$, either the sequence $\left\{f_{l}\right\}$ is uniformly bounded, or $\left\{\frac{1}{f_{l}}\right\}$ is uniformly bounded. By the $\mathbf{C}^{n}$-version of the Montel theorem: A family of uniformly bounded holomorphic functions in a domain of $\mathbf{C}^{n}$ is normal (see e.g. [3, p. 54]), we know that $\left\{f_{l}\right\}$ or $\left\{\frac{1}{f_{l}}\right\}$ is a normal family. In either case, we obtain a subsequence of $f_{n}$ that converges in $|z-\zeta|<\frac{R}{16}$. (Note that the limit function is allowed to be $\infty$ for a normal family.) This shows that $F$ is a normal family in $D$.

We conclude the paper by noting another consequence of Theorem 2: Any meromorphic solution $u$ of the eiconal and Fermat partial differential equations $\left(\frac{\partial u}{\partial z_{1}}\right)^{m}+\left(\frac{\partial u}{\partial z_{2}}\right)^{m}+\cdots+\left(\frac{\partial u}{\partial z_{n}}\right)^{m}=1$, or more generally, $a_{1}(z)\left(\frac{\partial u}{\partial z_{1}}\right)^{m}+a_{2}(z)\left(\frac{\partial u}{\partial z_{2}}\right)^{m}+$ $\cdots+a_{n}(z)\left(\frac{\partial u}{\partial z_{n}}\right)^{m}=b(z)$ in $\mathbf{C}^{n}$, where $m \geq 1$ is any positive integer and $b \not \equiv 0$, $a_{j}(1 \leq j \leq n)$ are polynomials, assumes each complex number without any exceptions. This is a Picard-type theorem. (The Picard theorem says that a non-constant meromorphic function in $\mathbf{C}$ assumes each complex number with possibly two exceptions.) The proof follows immediately from Theorem 2: If for some complex number $a$, the function $f:=u-a$, which is still a solution of the equation and thus not a constant, does not have any zeros in $\mathbf{C}^{n}$, then by Theorem 2 (taking $R=2 r$, we have that $T(r, f)=O\{\log r\}$, which implies that $f$ is a polynomial without zeros, and thus $f$ and then $u$ must be a constant, a contradiction. Similarly, a meromorphic solution $u$ of the above partial differential equation in the ball $|z|<R<+\infty$ with $T(r, u) \neq O\left\{\log \frac{1}{R-r}\right\}$ as $r \rightarrow R$ assumes each complex number without any exceptions. The condition $T(r, u) \neq O\left\{\log \frac{1}{R-r}\right\}$ as $r \rightarrow R$ cannot be dropped here. For example, $u=\left(z_{1}-1\right) \sin \frac{1}{1-z_{2}}$ is a solution of the equation $a_{1}(z)\left(\frac{\partial u}{\partial z_{1}}\right)^{2}+a_{2}(z)\left(\frac{\partial u}{\partial z_{2}}\right)^{2}=b(z)$ with $a_{1}=\left(z_{1}-1\right)^{2}, a_{2}=\left(1-z_{2}\right)^{4}$ and $b=\left(z_{1}-1\right)^{2}$. But, it is easy to check that this solution $u$ does not assume 0 in $|z|<R$ when $R$ is small.

In fact, a meromorphic function $f$ is called admissible in $|z|<R \leq+\infty$ if either $R=+\infty$ and $f$ is non-constant, or if $R<+\infty$ and $T(r, f) \neq O\left\{\log \frac{1}{R-r}\right\}$ as $r \rightarrow R$ (see [4, p. 42]). Thus, the above shows that an admissible meromorphic solution in $|z|<R \leq+\infty$ of the above partial differential equation assumes each complex number without any exceptions.

We refer to [7, [8 for some other results such as the structure of entire solutions of Fermat partial differential equations. 


\section{ACKNOWLedGement}

The author would like to thank the referee for valuable suggestions.

\section{REFERENCES}

[1] Berenstein, C.A. and Gay, R., Complex Variables, Springer-Verlag, New York, 1991. MR:1107514 (92f:30001)

[2] A. Biancofiore and W. Stoll, Another proof of the lemma of the logarithmic derivative in several complex variables, Ann. of Math. Stud., 100, Princeton Univ. Press, 1981, 29-45. MR627748 (83i:32007)

[3] R. Gunning, Introduction to Holomorphic Functions of Several Variables, Vol.1, Wadsworth, California, 1990. MR:1052649(92b:32001a)

[4] W. Hayman, Meromorphic functions, Clarendon Press, 1975. MR0164038 (29:1337)

[5] K. Hiong, Sur les fonctions holomorphes dont les dérivées admettent une valeur exceptionnelle, Ann. Ecole Norm. Sup. 72(1955), 165-197. MR0074525 (17:600h)

[6] S. Kobayashi, Hyperbolic Complex Spaces, Grundlehren der Mathematischen Wissenchaften, Vol. 318. Springer-Verlag, 1998. MR1635983 (99m:32026)

[7] B.Q. Li, On entire solutions of Fermat type partial differential equations, International Journal of Mathematics 15(2004), 473-485. MR2072090 (2005b:35023)

[8] B.Q. Li, Entire solutions of certain partial differential equations and factorization of partial derivatives, Transactions of AMS 357 (2005), 3169-3177. MR2135740 (2006a:32047)

[9] J. L. Schiff, Normal Families, Springer-Verlag, New York, 1993. MR.1211641 (94f:30046)

[10] W. Stoll, Holomorphic functions of finite order in several complex variables, Conference Board of the Mathematical Sciences, Regional Conference Series in Mathematics, no. 21, The American Mathematical Society, 1974. MR0350052 (50:2545)

[11] W. Stoll, Mehrfache Integrale auf komplexen Mannigfaltigkeiten, Math. Z. 57 (1952), 116-152. MR0051941 (14:550e)

[12] A. Vitter, The lemma of the logarithmic derivative in several complex variables, Duke Math. J. 44(1977), 89-104. MR0432924 (55:5903)

[13] L. Yang, Value Distribution Theory, Springer-Verlag, Berlin, 1993. MR1301781 (95h:30039)

[14] Z. Ye, On Nevanlinna's second main theorem in projective space, Invent. Math. 122 (1995), 475-507. MR.1359601 (96j:32030)

Department of Mathematics, Florida International University, Miami, Florida 33199

E-mail address: libaoqin@fiu.edu 